\title{
Unconventional diffusion of light in strongly localized open absorbing media
}

\author{
Li-Yi Zhao, ${ }^{1}$ Chu-Shun Tian, ${ }^{1}$ Zhao-Qing Zhang, ${ }^{2}$ and Xiang-Dong Zhang ${ }^{3}$ \\ ${ }^{1}$ Institute for Advanced Study, Tsinghua University, Beijing 100084, China \\ ${ }^{2}$ Department of Physics and Institute for Advanced Study, \\ Hong Kong University of Science and Technology, Clear Water Bay, Kowloon, Hong Kong \\ ${ }^{3}$ School of Physics, Beijing Institute of Technology, Beijing 100081, China
}

(Received 1 April 2013; revised manuscript received 15 August 2013; published 3 October 2013)

\begin{abstract}
Very recent experiments have discovered that localized light in strongly absorbing media displays intriguing diffusive phenomena. Here we develop a first-principles theory of light propagation in open media with arbitrary absorption strength and sample length. We show analytically that waves in localized open absorbing media exhibit highly unconventional diffusion. Specifically, wave energy transport follows the diffusion equation with the diffusion coefficient exhibiting spatial resolution. Most strikingly, despite that the system is controlled by two parameters - the ratio of the localization (absorption) length to the sample length — the spatially resolved diffusion coefficient displays novel single parameter scaling: It depends on the position in the sample via the returning probability. Our analytic predictions for this diffusion coefficient are confirmed by numerical simulations. In the strong absorption limit they agree well with the experimental results.
\end{abstract}

DOI: 10.1103/PhysRevB.88.155104

PACS number(s): 42.25.Dd, 71.23.An

In spite of microscopic complexity, classical electromagnetic waves (light) in infinite random media exhibit simple and universal behaviors on macroscopic scales. For example, the average intensity obeys a diffusion equation governed by the diffusion coefficient. The latter includes renormalization effects due to interference. When it vanishes light localization occurs. ${ }^{1,2}$ Yet, real experiments or devices usually deal with open media ${ }^{3-5}$ that have many profound differences from infinite media. For example, in open media wave transport cannot be fully suppressed, even disorders are strong. Can localized waves thereby exhibit a simple and universal diffusive behavior on macroscopic scales? This is a long-standing fundamental issue in localization physics (see Ref. 6 for a recent review). It is also of practical importance for considerable experiments especially on classical waves. ${ }^{3,4,7}$ For example, although it is possible to experimentally measure the distribution of intensity profile, to present and analyze it is difficult because the intensity profile depends on both the observation point and external sources. Instead, the (arithmetic) average of intensity profile can be easily obtained in experiments and be used to probe the important new physics appearing only in the localized regime such as resonant transmission. 5,8

Previous studies of electron conductance ${ }^{9,10}$ show that, in the localized regime, the geometrical average of the conductance exhibits a single-parameter scaling behavior but the arithmetic average does not, because the conductance distribution is very broad. Similarly, the average intensity is not a proper scaling variable in the localized regime. One might thereby expect that it would not exhibit any simple and universal behaviors on macroscopic scales. Such conjecture was also enforced by microwave experiments on a large ensemble of 10,000 disordered samples. ${ }^{4}$ There, the prevailing, albeit phenomenological, macroscopic diffusion model developed in Ref. 11 was found to completely fail to describe long-time transport of localized waves due to the fact that it cannot capture rare, long-lived, resonant transmission states. ${ }^{8}$ In fact, it is precisely due to the nonscaling nature of average intensity that has made the theoretical study of wave transport in localized open media notoriously difficult.

Surprisingly, an analytical result, ${ }^{12}$ found by using a firstprinciples theory ${ }^{6,13}$ and fully confirmed by numerical simulations, unveils a highly unconventional diffusive phenomenon in localized nonabsorbing open media. ${ }^{6,12}$ That is, the diffusion coefficient exhibits a novel scaling behavior, i.e., it depends on position in the sample via the returning probability. As a result of the novel scaling, the diffusion coefficient near boundaries is dominated by typical localized states while near the sample center by rare resonant transmission states. ${ }^{5}$ These analytical results allow us to calculate the average intensity profile as well as the average transmission via the spatially resolved diffusion coefficient. They show explicitly how rare resonant transmission states dominate the average transmission in strongly localized regime.

In this work, we show that the strong interplay between absorption and localization leads to even more interesting macroscopic diffusive phenomena. Strikingly, although the system is controlled by two parameters, namely the ratio of the localization (absorption) length to the sample length, the diffusion coefficient also exhibits a novel single parameter scaling. The analytical result for the diffusion coefficient is confirmed by numerical experiments. Compared to the nonabsorbing case, it is enhanced due to the suppression of localization effects by absorption, and the relative increase near the sample center is larger than near boundaries. For weak absorption, the spatial resolution of the diffusion coefficient overall is similar to that in the nonabsorbing case; for strong absorption, it exhibits a plateau deep inside the sample, in agreement with experimental results.

Main results and experimental relevance. Specifically, we consider propagation of light in quasi-one-dimensional open (uniformly) absorbing media of length $L$. This system is controlled by two dimensionless parameters, $\xi / L$ and $\xi_{a} / L$, where $\xi\left(\xi_{a}\right)$ is the localization (absorption) length. We find that-even in localized samples $(\xi / L \ll 1)$-waves exhibit macroscopic diffusion irrespective of the absorption rate $\gamma$. 
TABLE I. Behavior of $D(x)$ at $\xi \ll \xi_{a} \ll L$

\begin{tabular}{lc}
\hline \hline Region & $D(x) / D(0)$ \\
\hline$x \gtrsim \xi_{a} / 2$ & $e^{-\xi_{a} /(2 \xi)}$ \\
$\sqrt{\xi_{a} \xi} \ll x \ll \xi_{a} / 2$ & $e^{x^{2} /\left(\xi_{a} \xi\right)} e^{-x / \xi}$ \\
$\xi \lesssim x \ll \sqrt{\xi_{a} \xi}$ & $e^{-x / \xi}$ \\
$x \ll \xi$ & $1+c_{1}(x / \xi)+c_{2}(x / \xi)^{2}+\cdots$ \\
\hline \hline
\end{tabular}

More precisely, in the presence of a steady monochromatic light source located at $x^{\prime}$ the (average) intensity (namely energy density) profile, $\mathcal{Y}\left(x, x^{\prime}\right)$, obeys

$$
\left[\gamma-\partial_{x} D(x) \partial_{x}\right] \mathcal{Y}\left(x, x^{\prime}\right)=\delta\left(x-x^{\prime}\right) .
$$

It resembles the normal diffusion equation, but, the diffusion coefficient exhibits a number of anomalies. First of all, it has a spatial resolution, $D(x)$. This implies a local Fick's law. More precisely, the energy flux is given by $-D(x) \partial_{x} I(x)$, with $I(x)$ the wave density profile. Notice that $D(x)$ is an intrinsic quantity independent of the source and the wave density profile. It decreases monotonically from the boundary to the sample midpoint and varying over several orders. Most strikingly, despite that the system is controlled by two parameters, $D(x)$ exhibits a novel single parameter scaling,

$$
D(x) / D(0)=D_{\infty}[\lambda(x)],
$$

similar to the nonabsorbing medium case. Here, the scaling factor $\lambda(x)$ is proportional to the (static) returning probability depending on $x / L$ as well as $\xi / L$ and $\xi_{a} / L$, and the scaling function $D_{\infty}(\lambda)$ is the same as that of nonabsorbing media. [The explicit form of both $\lambda(x)$ and $D_{\infty}(\lambda)$ will be given below.] Bearing these anomalies and the analogy to the normal diffusion equation, Eq. (1) indicates highly unconventional diffusion of waves in open absorbing media. Furthermore, using Eq. (2), we can predict analytically the spatial resolution of the diffusion coefficient, which is confirmed by numerical experiments. We stress that our analytic results are very general: They are valid for arbitrary sample length and absorption strength and for both time-reversal (orthogonal symmetry) and broken time-reversal (unitary symmetry) systems. In addition, they are valid for both quasi- and strictly one-dimensional systems. In the former case, $\mathcal{Y}$ results from the integration over the cross section.

Recent experiments (both real and numerical) ${ }^{7,14,15}$ focus only on short samples and strong absorption, where resonant transmission states are unimportant. In this limiting case our analytic results are simplified and fully agree with the recent experimental findings there. Specifically, if absorption is strong such that $\xi_{a} / L \ll 1$, we find that $D(x)$ exhibits a plateau in the region $\min (x, L-x) \gtrsim \xi_{a}$. Moreover, the plateau value is determined by single parameter, $\xi_{a} / \xi$,

$$
D(L / 2) / D(0)=D_{\infty}\left(\xi_{a} / 2 \xi\right)
$$

(cf. the first line of Tables I and II). We stress that the plateau as well as Eq. (3) exists only if $\xi_{a} / L \ll 1$.

Origin of novel scaling. Since near the boundary, wave energy is easier to leak out of the medium (cf. dashed lines of Fig. 1), the returning probability, $\mathcal{Y}_{0}(x, x)$, is inhomogeneous in space (i.e., depending on $x$ ). As such, wave interference effects are inhomogeneous also. Near the boundary (deep
TABLE II. Behavior of $D(x)$ at $\xi_{a} \ll \xi \ll L$

\begin{tabular}{lc}
\hline \hline Region & $D(x) / D(0)$ \\
\hline$x \gtrsim \xi_{a} / 2$ & $1+c_{1}\left(\xi_{a} / 2 \xi\right)+c_{2}\left(\xi_{a} / 2 \xi\right)^{2}+\cdots$ \\
$x \ll \xi_{a} / 2$ & $1+c_{1}(x / \xi)+c_{2}(x / \xi)^{2}+\cdots$ \\
\hline \hline
\end{tabular}

inside the sample) they are weak (strong). For diffusive samples $\left(\xi / L \gg 1\right.$ where $\xi \sim \pi v S D_{0}{ }^{16}$ with $v$ the photon density of states and $S$ the cross-section area), $\mathcal{Y}_{0}(x, x)$ gives rise to an inhomogeneous (one-loop) weak localization correction $\sim \mathcal{O}\left[\mathcal{Y}_{0}(x, x) /(\pi \nu S)\right] \sim \mathcal{O}[\lambda(x)]$. For localized samples $(\xi / L \ll 1)$, as waves penetrate deeply into the sample optical paths easily return to its departure point causing more complicated wave interference. For example, two optical paths may take the same $n(=3$ in Fig. 1) loops as their routes and trace them with different orders. They thereby constructively interfere with each other. This leads to a weak localization correction $\sim \mathcal{O}\left[\lambda^{n}(x)\right]$. Therefore, $D(x)$ depends on $x / L$ (as well as $\xi / L$ and $\left.\xi_{a} / L\right)$ via the factor $\lambda(x)$, justifying Eq. (2).

Importantly, the optical paths in Fig. 1 may propagate in the same direction-clockwise or counterclockwise-during tracing each loop, suggesting that interference picture beyond what leads to usual one-loop weak localization does not necessarily require time-reversal symmetry. This is crucial to localization physics of systems with unitary symmetry. Therefore, the novel scaling is an intrinsic wave phenomenon unrelated to time-reversal symmetry.

First-principles theory. The derivations are largely parallel to those of nonabsorbing media. ${ }^{12,13}$ Therefore, we shall outline the key steps below with an emphasis on the key differences, and refer the readers to Ref. 6 for technical details. We first introduce the microscopic formalism valid for arbitrary dimensions. Consider the point-like source located at $\mathbf{r}^{\prime}$ inside the medium with the spectral decomposition $J_{\omega}\left(\mathbf{r}^{\prime}\right)$ ( $\omega$ being the wave angular frequency). The time-integrated intensity-upon disorder averaging-is given by ${ }^{17} I(\mathbf{r})=$ $\int \frac{d \omega}{\omega^{2}} v(\omega)\left|J_{\omega}\left(\mathbf{r}^{\prime}\right)\right|^{2} \mathcal{Y}\left(\mathbf{r}, \mathbf{r}^{\prime}\right)$. Here the (static) spatial correlation function $\mathcal{Y}\left(\mathbf{r}, \mathbf{r}^{\prime}\right)=\frac{\omega^{2}}{2 \pi \nu}\left\langle G_{\omega^{2}}^{A}\left(\mathbf{r}, \mathbf{r}^{\prime}\right) G_{\omega^{2}}^{R}\left(\mathbf{r}^{\prime}, \mathbf{r}\right)\right\rangle$, with $\langle\cdots\rangle$ standing for the disorder average and the advanced (retarded) Green function $G^{A}\left(G^{R}\right)$ defined as

$$
\left\{\nabla^{2}+\omega^{2}\left[1+\epsilon(\mathbf{r}) \pm i \epsilon^{\prime \prime}\right]\right\} G_{\omega^{2}}^{R, A}\left(\mathbf{r}, \mathbf{r}^{\prime}\right)=\delta\left(\mathbf{r}-\mathbf{r}^{\prime}\right),
$$

where the dielectric fluctuation, $\epsilon(\mathbf{r})$, is Gaussian, $\epsilon^{\prime \prime}>0$ causes uniform absorption, and the light velocity in the air is set to unity.

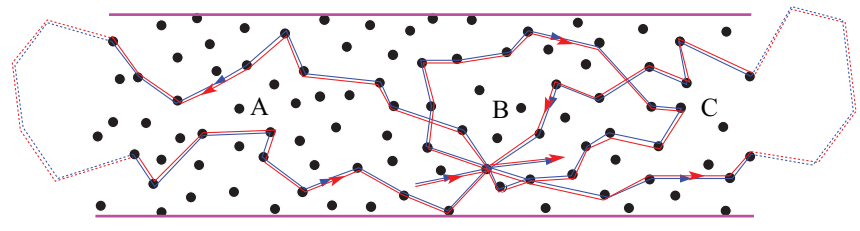

FIG. 1. (Color online) Examples of wave interference between two optical paths (red and blue) leading to the novel scaling behavior. The paths trace the three loops with different orders $(B \rightarrow A \rightarrow C$ for the red and $\mathrm{C} \rightarrow \mathrm{B} \rightarrow \mathrm{A}$ for the blue). Notice that these loops may not be complete (e.g., dashed parts of A and C) due to wave energy leakage. 
Then, it is a standard procedure to cast the above spatial correlation function in terms of the functional integral over a supermatrix field $Q(\mathbf{r})$,

$$
\mathcal{Y}\left(\mathbf{r}, \mathbf{r}^{\prime}\right)=\frac{\pi v}{128} \int D[Q] \operatorname{str}\left[A_{+} Q(\mathbf{r}) A_{-} Q\left(\mathbf{r}^{\prime}\right)\right] e^{-F[Q]} .
$$

Here, $A_{ \pm}$are some constant supermatrices and 'str' is the supertrace. ${ }^{6,16} F[Q]=\frac{\pi v}{8} \int d \mathbf{r} \operatorname{str}\left[D_{0}(\nabla Q)^{2}-2 \gamma \Lambda Q\right]$ differs from the nonabsorbing action ${ }^{6}$ in the second term accounting for the absorption. Notice that the (bare) diffusion coefficient $D_{0} \equiv D(0)$ and for $\epsilon^{\prime \prime} \ll 1$ the absorption rate $\gamma=\omega \epsilon^{\prime \prime} .{ }^{18}$ The supermatrix $Q=T^{-1} \Lambda T$, where $\Lambda$ is the so-called metallic saddle point and $T$ takes the value from the coset space of $U O S P(2,2 \mid 4) / U O S P(2 \mid 2) \otimes U O S P(2 \mid 2)$ for orthogonal symmetry and of $U(1,1 \mid 2) / U(1 \mid 1) \otimes U(1 \mid 1)$ for unitary symmetry. It is very important that if the boundary is transparent, the supermatrix field is fixed to be $\Lambda$ at the boundary. This reflects that on the boundary there is no wave energy accumulation. ${ }^{6,12,13}$

The microscopic formalism is simplified in quasi one dimension: $Q$ is homogeneous in the transverse plane; $F[Q]$ reduces to $\frac{\pi \nu S}{8} \int_{0}^{L} d x \operatorname{str}\left[D_{0}\left(\partial_{x} Q\right)^{2}-2 \gamma \Lambda Q\right]$ and the boundary condition to $Q(x=0)=Q(x=L)=\Lambda$. As a result, $\mathcal{Y}\left(\mathbf{r}, \mathbf{r}^{\prime}\right)$ depend only on the longitudinal coordinate. Integrating the cross section coordinates gives $\mathcal{Y}\left(x, x^{\prime}\right)$. To explicitly calculate the latter, we follow the procedures of Refs. 6 and 13. We find that $\mathcal{Y}\left(x, x^{\prime}\right)$ satisfies Eq. (1) with the boundary condition: $\mathcal{Y}\left(x=0, x^{\prime}\right)=\mathcal{Y}\left(x=L, x^{\prime}\right)=$ 0 . Furthermore, we find that the diffusion coefficient has a spatial resolution $D(x)$, and the latter is a functional of the factor $\lambda(x)=\mathcal{Y}_{0}\left(x, x^{\prime}=x\right) /(\pi v S)$. This justifies Eq. (2). $\mathcal{Y}_{0}\left(x, x^{\prime}\right)$ satisfies: $\left(\gamma-D_{0} \partial_{x}^{2}\right) \mathcal{Y}_{0}\left(x, x^{\prime}\right)=\delta\left(x-x^{\prime}\right), \mathcal{Y}_{0}(x=$ $\left.0, x^{\prime}\right)=\mathcal{Y}_{0}\left(x=L, x^{\prime}\right)=0$. Solving this equation we obtain

$$
\lambda(x)=\frac{\xi_{a}}{2 \xi} \frac{\cosh \left(L / \xi_{a}\right)-\cosh \left[(L-2 x) / \xi_{a}\right]}{\sinh \left(L / \xi_{a}\right)},
$$

where the diffusive absorption length $\xi_{a} \equiv \sqrt{D_{0} / \gamma}$.

For $\lambda \ll 1$, we find that the perturbative expansion of the scaling function $D_{\infty}(\lambda)$ is identical to that at $\gamma=0$. This implies that $D_{\infty}(\lambda)$ is the same as the one at $\gamma=0$. The latter has been found analytically and fully confirmed by numerical experiments, ${ }^{12}$ which has the following asymptotic form:

$$
D_{\infty}(\lambda) \sim \begin{cases}1+c_{1} \lambda+c_{2} \lambda^{2}+\cdots, & \lambda \ll 1, \\ e^{-\lambda,} & \lambda \gtrsim 1 .\end{cases}
$$

Notice that the coefficients $c_{i}$ depend on the system's symmetry. In particular, for orthogonal symmetry $c_{1}<0$, while for unitary symmetry $c_{1}=0, c_{2}<0$.

Evidence from numerical experiments. We perform numerical simulations of the (microscopic) Helmholtz equation. To this end we prepare a randomly layered medium. The relative permittivity in each layer is a random number with a real part uniformly distributed in the interval $[0.3,1.7]$. The imaginary part, $\epsilon^{\prime \prime}$, is assumed to be a constant. The random medium is embedded in the air background so that there exist no internal reflections when $\epsilon^{\prime \prime}$ vanishes. We launch a plane wave of frequency $\omega=1.65 a^{-1}$ into the system, where the sample length $L=200 a$ with $a$ the layer thickness, and use the standard transfer matrix method to calculate the intensity

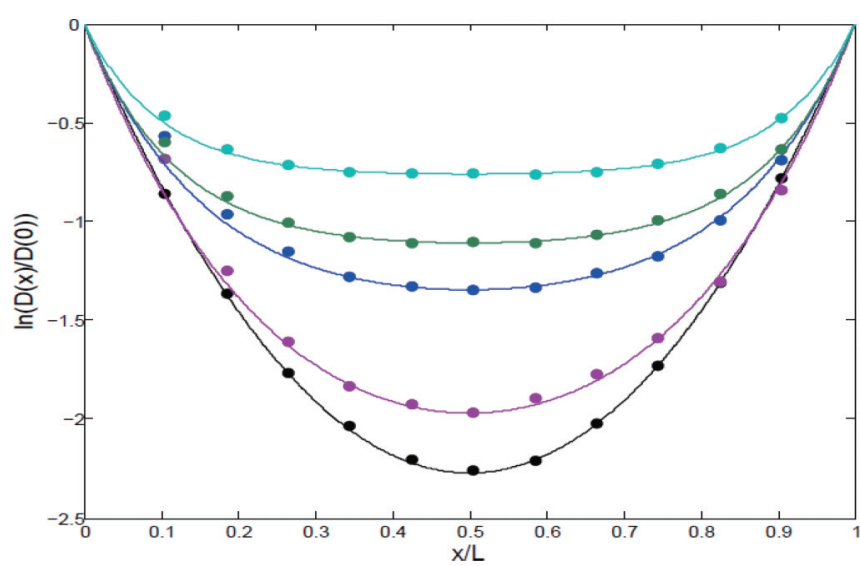

FIG. 2. (Color online) In localized open absorbing media the spatial resolution of the diffusion coefficient exhibits novel singleparameter scaling. Analytic predictions for $D(x)$ (solid lines) are in good agreement with the simulation results (circles). From bottom to top the absorption strength increases (see the text for the value of $\epsilon^{\prime \prime}$ as well as other parameters).

profile, $I_{\beta}(x)$, for each dielectric disorder configuration $\beta$. For a given value of $\epsilon^{\prime \prime}$, we calculate the ensemble-average intensity profile, $I(x) \equiv\left\langle I_{\beta}(x)\right\rangle$, of 5000000 disorder configurations. Then, by presuming Eq. (1), which gives $D(x)=$ $\left[-\gamma \int_{x}^{L} I\left(x^{\prime}\right) d x^{\prime}+\left.D(0) \partial_{x}\right|_{x=L} I(x)\right] / \partial_{x} I(x)$, we numerically obtain $D(x)$ from $I(x)$.

The results of simulations are shown in Fig. 2 for $\epsilon^{\prime \prime}=$ $0,1 \times 10^{-4}, 5 \times 10^{-4}, 1 \times 10^{-3}$, and $2 \times 10^{-3}$ (from bottom to top). First of all, in the nonabsorbing limit $\left(\epsilon^{\prime \prime}=0\right.$, the bottom curve) we recover the result of Ref. 12 for deeply localized (indeed, in this limit $L / \xi=200 a / 22 a \approx 9$ is large) samples. The effects of absorption become significant when $\xi_{a} / L \sim \mathcal{O}(1)$, i.e., $\epsilon^{\prime \prime} \approx \xi /\left(L^{2} \omega\right) \approx 3 \times 10^{-4}$. Having this estimation in mind, we may adjust the value of $\epsilon^{\prime \prime}$ to systematically explore the effects of absorption. The results are compared with the analytic predictions obtained from the novel single parameter scaling theory namely Eqs. (2), (6), and (7). As shown in Fig. 2, they are in good agreement. Now we present analytic results of $D(x)$ obtained from the developed first-principles theory.

$D(x)$ in deeply localized samples. It has been shown ${ }^{12}$ that for these samples $(\xi / L \ll 1)$ resonant transmission states play decisive roles in establishing the highly unconventional macroscopic diffusion of localized waves when absorption is absent. Due to long lifetime, these states are very sensitive to absorption. For weak absorption, $\xi_{a} / L \gg 1$, Eq. (6) reduces to $\lambda(x) \approx \frac{x(L-x)}{L \xi}\left\{1-\left[\frac{x(L-x)}{3 \xi_{a}^{2}}\right]\right\}$. The second line of Eq. (7) gives $D(x) \sim e^{-\lambda(x)}$ for $\lambda(x) \gg 1$. Thus, $D(x)$ enhances from its value in the nonabsorbing case, which is $\sim e^{-\frac{x(L-x)}{L \xi} 12}$, by a factor of $\sim \exp \left\{\frac{1}{3}\left[\frac{x(L-x)}{\xi_{a} \sqrt{L \xi}}\right]^{2}\right\}$. The latter increases monotonically from the boundary to the sample midpoint. Such inhomogeneous enhancement reflects that resonant transmission states are "killed" by absorption. Near the sample midpoint they have the longest lifetime and are most easily to be "killed," and this accounts for the strongest enhancement at the midpoint.

For strong absorption, $\xi_{a} / L \ll 1$, Eq. (6) reduces to $\lambda(x)=$ $\frac{\xi_{a}}{2 \xi}\left(1-e^{-2 x / \xi_{a}}\right)$ for $x \ll \xi_{a} / 2$ while to $\lambda(x) \approx \lambda(L / 2) \approx$ 


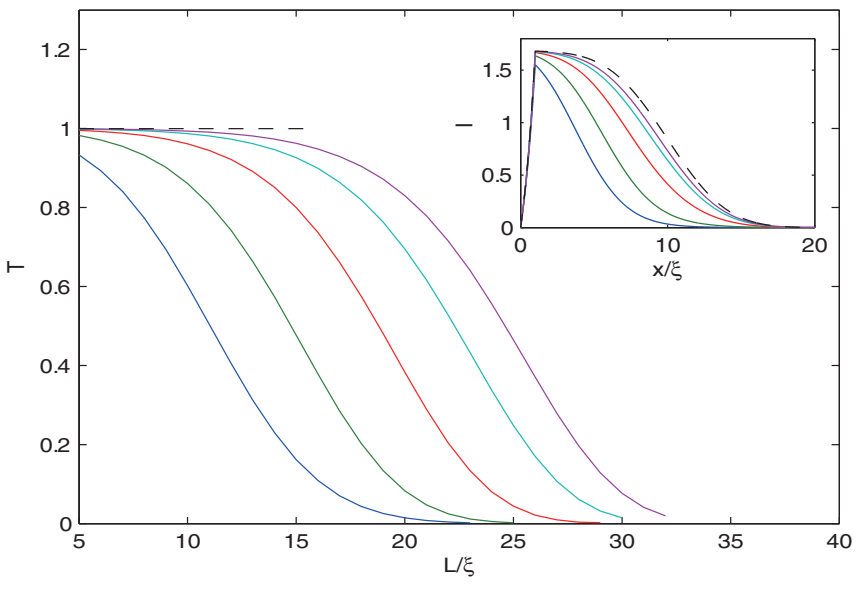

FIG. 3. (Color online) Effects of absorption on the average transmission $T(L)$, which is rescaled by that of nonabsorbing media $T_{0}(L)$. Inset: the corresponding intensity profiles for $L / \xi=20$. For the solid curves, the value of $\xi_{a} / \xi$ is $100,70,40,20$, and 10 from top to bottom, while for the dashed curve $\xi_{a} / \xi=\infty$.

$\xi_{a} /(2 \xi)$ for $x \gtrsim \xi_{a} / 2$. (We consider only $x \leqslant L / 2$ because of $D(x)=D(L-x)$.) Then, similar to discussions above we use Eq. (7) to find $D(x)$, and the results are summarized in Tables I and II. We see that $D(x)$ exhibits a plateau around the sample center. Strikingly, despite that the system is controlled by two parameters, $\xi / L$ and $\xi_{a} / L$, the plateau value depends on single parameter, $\xi_{a} / \xi$. More precisely, Eqs. (2) and (6) give Eq. (3). Moreover, from Eq. (7) we obtain the expression of $D(L / 2)$ for $\xi_{a} / 2 \xi \gg 1\left(\xi_{a} / 2 \xi \ll 1\right)$, which is given by the first line of Table I (II). The physical origin of the plateau can be understood from the fact that waves can only propagate a finite distance $\sim \xi_{a}$ in an absorbing medium, and the waves deep inside the medium cannot see the existence of open boundaries and resonant transmission states do not play a role here. (As such, deep inside the medium the boundary is not "seen" and resonance states play no roles.) Therefore, one might expect that the phenomenological model of Ref. 11 is valid, as observed in numerical experiments. ${ }^{14}$ Indeed, the first line of Table I was obtained a long time ago by using the field theory of infinite absorbing media. ${ }^{1}$

The second and third lines in Table I indicate a new scale, $\sqrt{\xi_{a} \xi}$. For $x \ll \sqrt{\xi_{a} \xi}, D(x)$ behaves essentially the same as that of semi-infinite nonabsorbing media, as shown in the third and fourth lines. The deviation starts at $x \sim \sqrt{\xi_{a} \xi}$ and has a physical explanation as follows. Light incident on the boundary effectively penetrates into the medium with a depth $\xi_{a}$. As such, the Lyapunov exponent (inverse localization length), $\bar{\gamma}$, fluctuates, following a distribution $\sim e^{-\frac{\xi a \xi}{4}\left(\bar{\gamma}-\xi^{-1}\right)^{2}} \cdot{ }^{9}$ Averaging $e^{-\bar{\gamma} x}$ with respect to this distribution, we recover the result given in the second line.

We can numerically calculate Eq. (1) to find the intensity profile, which allows us to study overall effects of absorption on transport. For simplicity, we focus on the one-dimensional case and place the source at $x^{\prime}=\xi$. Representative results for the intensity profile $I(x)$ are shown in the inset of Fig. 3 (for $L / \xi=20)$. Obviously, although the diffusivity, namely $D(x)$, increases with increasing absorption (decreasing $\xi_{a} / \xi$ ), the intensity profile is everywhere more and more suppressed. $I(x)$ thereby obtained gives the average transmission, i.e., $T(L)=-\left.D_{0} \partial_{x} I(x)\right|_{x=L}$, and the results are shown in the main panel of Fig. 3. Importantly, even for weak absorption (e.g., the $\xi_{a} / \xi=70$ curve), $T(L)$ behaves dramatically different from the average transmission of nonabsorbing media, $T_{0}(L)$. This can be attributed to the fact that the contributions of resonant transmission states to $T(L)$ are readily suppressed by absorption. The above results are also valid in quasi one dimensions. For $\xi_{a} \ll \xi \ll L$, upon substituting $D(x)$ (the first line of Table II) into Eq. (1) and solving the equation analytically, we find $T(L) \sim \exp \left(-\frac{L}{\xi_{a}}-\frac{L}{4 \xi}\right)$, in agreement with the result obtained from the random matrix theory. ${ }^{19}$

In summary, we present the first microscopic theory showing that in localized open absorbing media, waves display highly unconventional diffusion. First of all, the diffusion coefficient is inhomogeneous in space; most strikingly, despite that the system is controlled by two parameters $(\xi / L$ and $\left.\xi_{a} / L\right)$, it exhibits novel single-parameter scaling, namely Eq. (2). The analytic predictions for $D(x)$ are confirmed by numerical simulations. We emphasize that our theory is very general and, particularly, valid for arbitrary absorption strength. In the limiting case of strong absorption realized experimentally, ${ }^{7}$ our results agree well with experimental measurements. It is very interesting to generalize the present theory to the gained system ${ }^{20,21}$ in the future, and this may have direct applications in random lasers. ${ }^{22}$

Acknowledgments. We thank A. Z. Genack and A. G. Yamilov for useful discussions. This work is supported by the NSFC (Grant No. 11174174) and by the Tsinghua University Initiative Scientific Research Program (Grant No. 2011Z02151).
${ }^{1}$ S. John, Phys. Rev. Lett. 53, 2169 (1984).

${ }^{2}$ A. Lagendijk, B. van Tiggelen, and D. S. Wiersma, Physics Today 62, 24 (2009).

${ }^{3}$ J. Wang and A. Z. Genack, Nature (London) 471, 345 (2011).

${ }^{4}$ Z. Q. Zhang, A. A. Chabanov, S. K. Cheung, C. H. Wong, and A. Z. Genack, Phys. Rev. B 79, 144203 (2009).

${ }^{5}$ K. Yu. Bliokh, Yu. P. Bliokh, V. Freilikher, S. Savel'ev, and F. Nori, Rev. Mod. Phys. 80, 1201 (2008).

${ }^{6}$ C. Tian, Physica E 49, 124 (2013).

${ }^{7}$ A. G. Yamilov, R. Sarma, B. Redding, B. Payne, H. Noh, and H. Cao, arXiv:1303.3244.
${ }^{8}$ I. M. Lifshits, S. A. Gredeskul, and L. A. Pastur, Introduction to the Theory of Disordered Systems (Wiley, New York, 1988); I. M. Lifshits and V. Y. Kirpichenkov, Sov. Phys. JETP 50, 499 (1979).

${ }^{9}$ P. W. Anderson, D. J. Thouless, E. Abrahams, and D. S. Fisher, Phys. Rev. B 22, 3519 (1980).

${ }^{10}$ A. Cohen, Y. Roth, and B. Shapiro, Phys. Rev. B 38, 12125 (1988), and references therein.

${ }^{11}$ B. A. van Tiggelen, A. Lagendijk, and D. S. Wiersma, Phys. Rev. Lett. 84, 4333 (2000).

${ }^{12}$ C. S. Tian, S. K. Cheung, and Z. Q. Zhang, Phys. Rev. Lett. 105, 263905 (2010) 
${ }^{13}$ C. Tian, Phys. Rev. B 77, 064205 (2008).

${ }^{14}$ A. G. Yamilov and B. Payne, Optics Express 21, 11688 (2013).

${ }^{15}$ B. Payne, A. Yamilov, and S. E. Skipetrov, Phys. Rev. B 82, 024205 (2010).

${ }^{16}$ K. B. Efetov, Supersymmetry in Disorder and Chaos (Cambridge University Press, Cambridge, England, 1997).

${ }^{17}$ V. Ya. Chernyak, K. I. Grigoshin, E. I. Ogievetsky, and V. M. Agranovich, Solid State Commun. 84, 209 (1992).
${ }^{18}$ For $\epsilon^{\prime \prime} \sim \mathcal{O}(1)$ or in strictly one dimensions, both $D_{0}$ and $\gamma$ strongly depend on $\epsilon^{\prime \prime}$. However, the detailed forms are unimportant for the present work, and therefore we shall not discuss this issue further. ${ }^{19}$ P. W. Brouwer, Phys. Rev. B 57, 10526 (1998).

${ }^{20}$ Z. Q. Zhang, Phys. Rev. B 52, 7960 (1995).

${ }^{21}$ P. Pradhan and N. Kumar, Phys. Rev. B 50, 9644 (1994).

${ }^{22}$ H. Cao, Y. G. Zhao, S. T. Ho, E. W. Seelig, Q. H. Wang, and R. P. H. Chang, Phys. Rev. Lett. 82, 2278 (1999). 